\title{
Hierarchical Survivable Network Design Problems
}

\author{
Inmaculada Rodríguez-Martín ${ }^{1,2}$ \\ Juan-José Salazar-González ${ }^{3}$ \\ DMEIO \\ University of La Laguna \\ Tenerife, Spain \\ Hande Yaman ${ }^{4}$ \\ Department of Industrial Engineering \\ Bilkent University \\ Ankara, Turkey
}

\begin{abstract}
We address the problem of designing two-level networks protected against single edge failures. A set of nodes must be partitioned into terminals and hubs, hubs must be connected through a backbone network, and terminals must be assigned to hubs and connected to them through access networks, being the objective to minimize the total cost. We consider two survivable structures, two-edge connected (2EC) networks and rings, in both levels of the network. We present an integer programming formulation for these problems, solve them using a branch-and-cut algorithm, and show some computational results.
\end{abstract}

Keywords: Network design, survivability, branch-and-cut. 


\section{Introduction}

We study several two level network design problems with survivability requirements in both levels. In a typical two level network, the upper level is called the backbone network and connects the hubs (concentrators, switches, multiplexers) among themselves, and the lower level networks are called access networks and they connect the users to hubs. Klincewicz [12] uses the notation "backbone structure/access structure" to specify the structure of a two level network. We are interested in designing two level networks that have protection against a single edge failure, i.e., we assume that at most one edge can fail at a time. We consider two survivable network structures, namely, 2-edge connected (2EC) networks and rings, in both levels of the network. As a result, we study the design problems associated with four different networks: $2 \mathrm{EC} / 2 \mathrm{EC}, 2 \mathrm{EC} /$ ring, ring/2EC and ring/ring networks.

The literature on the design of survivable networks has grown over the last years. However, most studies on survivable network design problems consider a single layer of the network (see the reviews by Grötschel et al. [9] and Kerivin and Mahjoub [11]). There are few studies that consider the design of two level networks with survivability requirements in both levels. The majority of such studies are on designing ring/ring networks (Thomadsen and Stidsen [19], Carroll and Mc Garraghy [3]), and most of the approaches proposed are heuristic approaches (Shi and Fonseca [17], Balakrishnan et al. [1]). The contribution of the present paper is to propose formulations and exact solution methods for the two level survivable network design problem where both rings and 2-edge connected networks are used to ensure survivability.

We can find many related problems in the field of network design. Fortz and Labbé [5] and Fortz et al. [6,7,8] study the design of networks with bounded rings. Magnanti and Raghavan [14] and Balakrishnan et al. [2] consider different variants of two-edge connected networks. Soriano et al. [18] and Caserta et al. [4] treat the design of survivable telecommunications networks based on ring structures. Finally, as examples of other types of two-level network design problems, we mention the ring/chain problem studied by Lee and Kon [13] and the capacitated ring/tree problem studied by Hill and Voß [10].

\footnotetext{
1 This work has been supported by "Ministerio de Educación y Ciencia", Spain (research project MTM2012-36163-C06-01). The research of the third author is supported by the Turkish Academy of Sciences.

2 Email: irguez@ull.es

3 Email: jjsalaza@ull.es

4 Email: hyaman@bilkent.edu.tr
} 


\section{Integer Linear Programming Models}

Let $V=\{0,1, \ldots, n\}$ be the set of nodes where node 0 stands for the root. We denote by $f_{j}$ the cost of installing a hub at node $j \in V$. Let $E=\{\{i, j\}: i, j \in$ $V, i<j\}$. We denote the costs of installing a backbone link and an access link on edge $e \in E$ with $b_{e}$ and $a_{e}$, respectively. The aim of the problem is to partition the set of nodes $V$ into disjoint subsets, each with at most $q$ nodes, choose one node from each subset to locate a hub and connect the hubs and the subsets with survivable networks at minimum cost.

Let $G=(V, E)$. For $S \subseteq V$, let $\delta(S)$ be the set of edges with one endpoint in $S$ and $E(S)$ be the set of edges with both endpoints in set $S$. When $S$ is a singleton, i.e., $S=\{i\}$, we use $\delta(i)$ for $\delta(\{i\})$.

We define $z_{i j}$ to be 1 if node $i \in V$ is assigned to hub $j \in V$ and to be 0 otherwise. Node $j$ is a hub if $z_{j j}$ is 1 . In addition, we define $x_{e}$ to be 1 if edge $e \in E$ is used in an access network and 0 otherwise and $y_{e}$ to be 1 if edge $e$ is used in the backbone network and 0 otherwise. For $E^{\prime} \subseteq E, x\left(E^{\prime}\right)=\sum_{e \in E^{\prime}} x_{e}$ and $y\left(E^{\prime}\right)=\sum_{e \in E^{\prime}} y_{e}$.

The 2EC/2EC design problem can be modeled as follows:

$$
\begin{array}{ll}
\min & \sum_{j \in V} f_{j} z_{j j}+\sum_{e \in E} a_{e} x_{e}+\sum_{e \in E} b_{e} y_{e} \\
\text { s.t. } & \sum_{j \in V} z_{i j}=1 \quad \forall i \in V, \\
& \sum_{i \in V} z_{i j} \leq q z_{j j} \quad \forall j \in V, \\
& z_{00}=1, \\
& z_{i j}+y_{e} \leq z_{j j} \quad \forall i, j \in V: e=\{i, j\} \in E, \\
& y(\delta(S)) \geq 2 \sum_{j \in S} z_{i j} \quad \forall S \subseteq V \backslash\{0\}, i \in S, \\
& x(\delta(S)) \geq 2 \sum_{j \in V \backslash S} z_{i j} \quad \forall S \subset V, i \in S, \\
& x_{i i^{\prime}}+z_{i j}+z_{i^{\prime} j^{\prime}} \leq 2 \quad \forall\left\{i, i^{\prime}\right\} \in E, j, j^{\prime} \in V, j \neq j^{\prime}, \\
& x_{e}, y_{e} \in\{0,1\} \quad \forall e \in E, \\
& z_{i j} \in\{0,1\} \quad \forall i, j \in V .
\end{array}
$$

The objective function is the sum of the cost of locating hubs and the cost of installing access and backbone links. Constraints (2) ensure that each node is either a hub or it is assigned to another hub. Constraints (3) are capacity 
constraints that limit the number of nodes assigned to a hub by $q$. They also ensure that no node is assigned to a nonhub node. Constraint (4) forces the root node to be a hub. If an edge is used in the backbone network, then due to constraints (5), both endpoints should be hubs. Constraints (6) impose 2-edge connectedness of the backbone network. If node $i$ is a hub or if it is assigned to a hub node in set $S$, then there exists at least one hub in set $S$ and the constraint asks for at least two backbone edges on the cut $\delta(S)$ since the root is in $V \backslash S$. Similarly, constraints (7) ensure 2-edge connectedness of the access networks. If node $i \in S$ is allocated to a hub node in $V \backslash S$ then there should be at least two access links between $S$ and $V \backslash S$. Constraints (8) make sure that if the access link $\left\{i, i^{\prime}\right\}$ is used then $i$ and $i^{\prime}$ are allocated to the same hub. Finally, constraints (9) and (10) are variable restrictions.

To model $2 \mathrm{EC} / \mathrm{ring}$ and ring/ring design problems, we add the degree constraints

$$
x(\delta(i)) \leq 2 \quad \forall i \in V .
$$

We can not impose (11) to be an equation because not all nodes have to be in an access network. Similarly, to model ring/2EC and ring/ring design problems, we add the constraints

$$
y(\delta(j))=2 z_{j j} \quad \forall j \in V,
$$

to make sure that each hub node has degree two in the backbone network.

\section{$3 \quad$ Valid Inequalities}

Rodríguez-Martín et al. [16] propose a family of valid inequalities that dominate constraints (8). Let $\left\{i, i^{\prime}\right\} \in E, S \subset V$ such that $i \in S$ and $i^{\prime} \in V \backslash S$. The inequality

$$
x_{i i^{\prime}} \leq \sum_{j \in V \backslash S} z_{i j}+\sum_{j^{\prime} \in S} z_{i^{\prime} j^{\prime}}
$$

is valid for $2 \mathrm{EC} / 2 \mathrm{EC}, 2 \mathrm{EC} /$ ring, ring/2EC and ring/ring problems.

Let $S \subset V$. The following inequalities used in [16] are also valid for our 
problems:

$$
\begin{aligned}
& x(\delta(S)) \geq 2\left(\left\lceil\frac{|S|}{q}\right\rceil-\sum_{i \in S} z_{i i}\right), \\
& x(\delta(S)) \geq 2\left(\frac{\sum_{i \in S} \sum_{j \in V} z_{i j}+x(\delta(S)) / 2}{q}-\sum_{i \in S} z_{i i}\right), \\
& x(\delta(S)) \geq 2\left(\sum_{j \in V \backslash S} z_{i j}+\sum_{j \in S} z_{i^{\prime} j}\right) \quad i \in S, i^{\prime} \in V \backslash S .
\end{aligned}
$$

\section{Computational Results}

We devised a branch-and-cut algorithm using the model and the results presented above. We coded it in C++ using the CPLEX 12.5 mixed integer linear programming solver. All the experiments were done on a personal computer with a processor Intel Core i7 CPU at $3.4 \mathrm{GHz}$ and $16 \mathrm{~GB}$ of RAM.

The behavior of the algorithm was tested on randomly generated instances and also on instances from the SNDlib [15], though the latter are not complete graphs and this implies code modifications. For the sake of brevity, we only report here results for one instance of each of those sets. In particular, Table 1 shows the results obtained for an instance with 20 nodes. For each edge $e \in E$ we generate a random figure $c_{e} \in[1,100]$, then the access and backbone costs for that edge are defined as $a_{e}=c_{e}$ and $b_{e}=4 c_{e}$ respectively. Table 2 shows the results for instance france from the SNDlib. This is an instance with 25 nodes and 45 undirected links with fixed charge costs. For each edge, the access cost is set equal to the fixed cost of that edge, and the backbone cost is set to four times that value. For both the random and the SNDlib instances, the hub costs $f_{j}$ are randomly generated in [500,1000].

For each instance we solved the four problems given by the different possible combinations of network topologies, and for each combination we tried three capacity values $(q:=\{\lceil n / 3\rceil,\lceil n / 4\rceil,\lceil n / 5\rceil\})$. The column headings in Tables 1 and 2 stand for the backbone/access networks' configurations, the capacity of the hubs, the optimal solution cost, the number of hubs in the optimal solution, the relative error, in percentage, between the lower bound at the end of the root node and the optimal cost, the number of nodes in the branch-and-cut search tree, and the total cpu time.

We observe that all problems are solved to optimality except the ring/ring and ring/2EC versions of the france instance, which turn out to be infeasi- 


\begin{tabular}{cc|ccccc}
\hline & $q$ & opt & nHubs & r-gap & nodes & cpu \\
\hline ring/ring & $\lceil n / 3\rceil$ & 2858 & 3 & 99.77 & 242 & 3.14 \\
& $\lceil n / 4\rceil$ & 3518 & 4 & 98.46 & 159 & 5.18 \\
& $\lceil n / 5\rceil$ & 4272 & 5 & 97.35 & 402 & 21.54 \\
\hline ring/2EC & $\lceil n / 3\rceil$ & 2855 & 3 & 99.27 & 171 & 1.08 \\
& $\lceil n / 4\rceil$ & 3518 & 4 & 98.17 & 347 & 8.56 \\
& $\lceil n / 5\rceil$ & 4272 & 5 & 97.25 & 779 & 59.16 \\
\hline \multirow{2}{*}{$2 \mathrm{EC} / \mathrm{ring}$} & $\lceil n / 3\rceil$ & 2858 & 3 & 95.20 & 277 & 24.15 \\
& $\lceil n / 4\rceil$ & 3518 & 4 & 97.44 & 487 & 28.55 \\
& $\lceil n / 5\rceil$ & 4272 & 5 & 96.84 & 1660 & 189.73 \\
\hline \multirow{2}{*}{$2 \mathrm{EC} / 2 \mathrm{EC}$} & $\lceil n / 3\rceil$ & 2855 & 3 & 94.72 & 122 & 9.52 \\
& $\lceil n / 4\rceil$ & 3518 & 4 & 97.10 & 494 & 15.37 \\
& $\lceil n / 5\rceil$ & 4272 & 5 & 96.69 & 961 & 86.80 \\
\hline
\end{tabular}

Table 1

Results for a random instance with $n=20$

ble. The tables also show the influence of the capacity on the computational performance.

\section{Conclusions and Outlook}

We have presented a MIP formulation and valid inequalities for a complex twolevel survivable network design problem. This formulation models with minor modifications the four possible combinations of the two topologies considered (rings and two-edge connected networks). We have designed a branch-and-cut algorithm and we show some preliminary computational results. For future studies, we hope to find new families of valid inequalities that serve to improve the performance of the branch-and-cut, so that we can tackle larger instances. 


\begin{tabular}{cc|ccccc}
\hline & $q$ & opt & nHubs & r-gap & nodes & cpu \\
\hline ring/ring & $\lceil n / 3\rceil$ & infeas. & & & & \\
& $\lceil n / 4\rceil$ & infeas. & & & & \\
& $\lceil n / 5\rceil$ & infeas. & & & & \\
\hline ring/2EC & $\lceil n / 3\rceil$ & infeas. & & & & \\
& $\lceil n / 4\rceil$ & infeas. & & & & \\
& $\lceil n / 5\rceil$ & infeas. & & & & \\
\hline $2 \mathrm{EC} /$ ring & $\lceil n / 3\rceil$ & 127879 & 10 & 86.10 & 7 & 7.50 \\
& $\lceil n / 4\rceil$ & 127879 & 10 & 87.91 & 4 & 6.63 \\
& $\lceil n / 5\rceil$ & 149968 & 16 & 73.54 & 57 & 37.86 \\
\hline \multirow{2}{*}{$2 \mathrm{EC} / 2 \mathrm{EC}$} & $\lceil n / 3\rceil$ & 119950 & 9 & 54.83 & 68 & 17.55 \\
& $\lceil n / 4\rceil$ & 127542 & 10 & 58.66 & 86 & 28.70 \\
& $\lceil n / 5\rceil$ & 149968 & 16 & 56.36 & 99 & 23.49 \\
\hline
\end{tabular}

Table 2

Results for SNDlib france instance $(n=25)$

\section{References}

[1] Balakrishnan, A., T.L. Magnanti, P. Mirchandani. Designing hierarchical survivable networks. Oper. Res. 46 (1998), 116-136.

[2] Balakrishnan, A., P. Mirchandani, H.P. Natarajan. Connectivity upgrade models for survivable network design. Oper. Res. 57 (2009) 170-186.

[3] Carroll, P., S. McGarraghy. A decomposition algorithm for the ring spur assignment problem. Intl. Trans. in Op. Res. 20 (2013), 119-139.

[4] Caserta, M., S. Schwarze, S. Voß. Developing a ring-based optical network structure with glass-through nodes. Journal of Telecommunications and Information Technology 2 (2012), 11-18.

[5] Fortz, B., M. Labbé. Two-connected networks with rings of bounded cardinality. Computational Optimization and Applications 27 (2004) 123-148.

[6] Fortz, B., M. Labbé, F. Maffioli. Solving the two-connected network with bounded meshes problem. Operations Research 48 (2000) 866-877. 
[7] Fortz, B., A.R. Mahjoub, S. McCormick, P. Pesneau. Two-edge connected subgraphs with bounded rings: polyhedral results and branch-and-cut. Mathematical Programming 105 (2006) 85-111.

[8] Fortz, B., P. Soriano, C. Wynants. A tabu search algorithm for self-healing ring network design. European Journal of Operational Research 151 (2003) 280-295.

[9] Grötschel, M., C.L. Monma, M. Stoer. "Design of survivable communications networks". M. O. Ball, T. L. Magnanti, C. L. Monma, G. L. Nemhauser, eds. Network Models. North-Holland, Amsterdam. 617-671. 1995.

[10] Hill, A., S. Voß. Generalized local branching heuristics and the capacitated ring tree problem. University of Antwerp, Faculty of Applied Economics, research paper 2014-020, 2014.

[11] Kerivin, H., A.R. Mahjoub. Design of survivable networks: a survey. Networks 46 (2005), 1-21.

[12] Klincewicz, J.G. Hub location in backbone/tributary network design: a review. Location Sci. 6 (1998), 307-335.

[13] Lee, C.Y., S.J. Koh. Design of the minimum cost ring-chain network with dualhoming survivability: a tabu search approach. Comp. Oper. Res. (1997) 24 883897.

[14] Magnanti, T.L., S. Raghavan. Strong formulations for network design problems with connectivity requirements. Networks 45 (2005) 61-79.

[15] Orlowski, S., R. Wessäly, M. Piòro, A. Tomaszewski. SNDlib 1.0 - Survivable Network Design Library. Networks 55 (2010), 276286

[16] Rodríguez-Martín, I., J-J. Salazar-González, H. Yaman. A Branch-and-cut Algorithm for the Hub Location and Routing Problem. Computers \& Operations Research 50 (2014), 161-174

[17] Shi, J.J, J.P. Fonseka. Hierarchical Self-Healing Rings. IEEE/ACM Transactions on Networking, 3 (1995), 690-697.

[18] Soriano, P., C. Wynants, R Seguin, M. Labbé, M. Gendreau, B. Fortz. Design and Dimensioning of Survivable SDH/Sonet Networks. B. Sanso and P. Sariano, eds. Telecommunications Network Planning. Springer (1999) 147-167.

[19] Thomadsen, T., T. Stidsen.Hierarchical ring network design using branch-andprice. Telecomm. Sys. 29 (2005), 61-76. 